Review

\title{
Review of Display Technologies Focusing on Power Consumption
}

\author{
María Rodríguez Fernández ${ }^{1, \dagger}$, Eduardo Zalama Casanova ${ }^{2, *}$ and Ignacio González Alonso ${ }^{3, \dagger}$ \\ 1 Department of Systems Engineering and Automatic Control, University of Valladolid, \\ Paseo del Cauce S/N, 47011 Valladolid, Spain; E-Mail: maria.rodriguez.fernandez@gmail.com \\ 2 Instituto de las Tecnologías Avanzadas de la Producción, University of Valladolid, \\ Paseo del Cauce S/N, 47011 Valladolid, Spain \\ 3 Department of Computer Science, University of Oviedo, C/González Gutiérrez Quirós, \\ 33600 Mieres, Spain; E-Mail: gonzalezaloignacio@uniovi.es
}

$\dagger$ These authors contributed equally to this work.

* Author to whom correspondence should be addressed; E-Mail: ezalama@eii.uva.es; Tel.: +34-659-782-534.

Academic Editor: Marc A. Rosen

Received: 16 June 2015 / Accepted: 4 August 2015 / Published: 11 August 2015

\begin{abstract}
This paper provides an overview of the main manufacturing technologies of displays, focusing on those with low and ultra-low levels of power consumption, which make them suitable for current societal needs. Considering the typified value obtained from the manufacturer's specifications, four technologies - Liquid Crystal Displays, electronic paper, Organic Light-Emitting Display and Electroluminescent Displays - were selected in a first iteration. For each of them, several features, including size and brightness, were assessed in order to ascertain possible proportional relationships with the rate of consumption. To normalize the comparison between different display types, relative units such as the surface power density and the display frontal intensity efficiency were proposed. Organic light-emitting display had the best results in terms of power density for small display sizes. For larger sizes, it performs less satisfactorily than Liquid Crystal Displays in terms of energy efficiency.
\end{abstract}

Keywords: energy efficiency; power consumption; Liquid Crystal Display; Organic Light-Emitting Display; Electroluminescent Display; electronic paper 


\section{Introduction}

The world as a whole is currently in an overshoot condition. Population size and economic growth contribute significantly to this situation [1]. Specifically, the steady growth in the use of personal portable devices during the past decade - driven by the development of mobile telecommunications and the incorporation of personal digital assistant devices and electronic books into the market-has considerably increased the per person carbon footprint [2]. The International Energy Agency (IEA), which monitors the demand and supply of energy worldwide, aims to reduce the global consumption level to an acceptable limit. In order to do so, the IEA is calling for more efficient technologies for the aforementioned devices to be manufactured. In the Energy Technology Perspectives Study, the IEA points out that technologies can and must play an integral role in transforming the energy system to reduce greenhouse gases [3].

Leaving aside the environmental reasons to reduce power consumption, the demand for power in electronic devices has also increased, especially those with limited battery lifespan that need to reduce their rates of power consumption for optimal performance. According to several studies [4,5], one of the components with the highest percentage of total energy consumption, and therefore a suitable candidate for improvement, is the display. Traditionally, the reduction of $\mathrm{t}$ display energy consumption in electronic devices was done through methods that often relied on shutting off the display during inactive periods [6] or adjusting the luminance of the screens with light-based automatic brightness control (LABC) [7] methods. Due to current market conditions, the focus on improvement needs to shift to make the device itself more power efficient, through the use of display technologies with higher levels of energy efficiency.

The aim of this review is to identify which display technologies are most appropriate in terms of power consumption. The displays can be classified according to different approaches, for instance regarding the way they create the image, their size, the maturity of the technology, etc. [8], but to the best of our knowledge, the power consumption is rarely taken into account in any of them as a parameter to make a difference between display technologies.

Firstly, in the next section, the scope of the study is outlined. In Section 3, the main features influencing in the consumption of a display are explained in order to be understandable in Section 4, where the specific advantages of those features for some specific display modules are listed. Section 5 includes the analysis, comparison and discussion of the mentioned data. Applications and trends are detailed in Section 6. Finally, conclusions will be drawn and future work suggested.

\section{Scope of the Study}

Many display modules of different technologies coexist and compete for their share in the market. Due to the diversity in sizes and brands, the establishment of a standardized method that enables a reliable power consumption comparison between them has been considered relevant.

The International Committee for Display Metrology aims to solve the need of the display industry and research institutions for having a single reference standard on how to measure and characterize displays. As a result, the Information Display Measurements Standard (IDMS) [9] has been published by the Society for Information Display [10], replacing the previous Flat Panel Measurements (FPDM) standard. 
Power consumption is defined by the IDMS as the power needed by the Device under Test (DuT) to reach its full operation. The components to be measured depend on the technology used to develop the display. For example, the power required for the backlight in LCD is part of the display power, since the DuT is not functional without it. As the power is additive, the total power of the device is the sum of all individual powers, including the backlight with inverter where applicable, the panel and any other incidental power from the elements needed to run the display.

In order to give a relative value to the power consumed by a display, different metrics can be used, such as the Environment Cost of Ownership (ECO) [11] for the Life Cycle Assessment (LCA) [12], that each look at the environmental impact of the technology. According to Vaclav Smil, the power density, which will be used in this work, is one of the most revealing variables in energetics for assessing consumption [13]. Following this approach, displays can be divided into high, medium, low and ultralow power consumption items (the proposed range values for each category are listed in Table 1).

A complete classification of the technologies is shown in the Graphical Abstract. For each one, the power consumption density of ten different commercialized display modules was analyzed, extracting their technical specifications from the manufacturer's datasheet. The results revealed that some displays show a remarkable increase in power consumption with size. Therefore, it was concluded that the size could be a second criterion for assessing display technologies.

In the field of display development, which is strongly linked to the path set by manufacturers, the Japan Displays Inc. consortium [14], founded in 2012 (Sony Mobile, Toshiba Mobile and Hitachi displays) is a reference in the industry. There are also other interesting initiatives, such as the German Flat Panel Display Forum (DFF) platform [15], founded in 2000 and composed of more than 65 companies and institutions of the display community. They deal with relevant matters to the field, like future technology trends or market monopolies. According to market research [16], the market share of displays with reduced dimensions has been duplicated in the last five years and it is expected to continue growing over the next seven years, especially due to head-mounted displays. This, and the fact that power consumption increases with the size, were the main drivers in reducing the scope of this article. Additionally, not all the display technologies are manufactured in all sizes, due to intrinsic restrictions or market directions.

The Cathode Ray Tube (CRT), although it has been used for decades, requires at least twice the power of a Liquid Crystal Display (LCD) [17], and only remains present in specific graphic-related fields, due to its higher color fidelity, contrast, and wide viewing angle. Field Emission Displays (FED) follows the idea of CRT, but uses millions of electron guns instead of only one [18]. Different field-emission electron sources can be used, including carbon nanotube Field Emission Displays (CNT-FED) [19] and Ballistic Electron Surface Emitting Display (BSD) [20], which are now being actively developed. This technology could offer slim, high quality for all sizes of display. Therefore, they are expected to be low-power displays, as little or no power is consumed in generating electrons. Motorola and Samsung, among others, are supporting this technology and they have already created some successful prototypes, producing high resolution images like CRT without the bulky appearance. Another closely related technology is Surface-conduction Electron-emitter (SED) [21], differing primarily in the details of the electron emitters.

Plasma Display Panel (PDP) is not economically profitable to be manufactured in small sizes [22] and nor is its subtype Alternate Lighting of Surfaces (ALiS). Digital Light Processing (DLP) is a system 
that uses an optical semiconductor device developed by Texas Instruments in 1987. The device, known as the Digital Mirror Device (DMD chip) is essentially a very precise light switch made up of millions of microscopic mirrors that, combined with a digital video or graphic signal, a light source, and a projection lens, can reflect an all-digital image onto any surface. DLP technology can generate large, bright projections with high contrast ratios on screens with up to 35 trillion colors. Other promising technology is Laser Phosphor Display (LPD), still in the development phase, hence numeric values cannot be provided due to the lack of available information on existing products. Thus, the comparison carried out in this study will be more reliable if the aforementioned categories and subcategories are left out.

In summation, the final chosen parameters for module classification are size and power consumption density. The size categories into which the modules can be classified are: microdisplays, typically not more than $25 \mathrm{~mm}$ (1 inch) image diagonal [23], small-sized (diagonal less than 7 inches), medium-sized (from 7 inches to 20) and large (more than 20 inches). The power consumption density categories are established in Table 1.

Table 1. Considered power density consumption ranges.

\begin{tabular}{cc}
\hline Power Consumption Level & Power density $\left(\mathbf{m W} / \mathbf{c m}^{2}\right)$ \\
\hline High & $>250$ \\
Medium & $100-250$ \\
Low & $10-100$ \\
Ultra-low & $0-10$ \\
\hline
\end{tabular}

Recalling the objectives set out in the introduction section, the scope of this work will cover the low-power and ultra-low power small-sized displays. The dotted line of Figure 1 limits the technologies that fulfill these requirements and will be analyzed further.

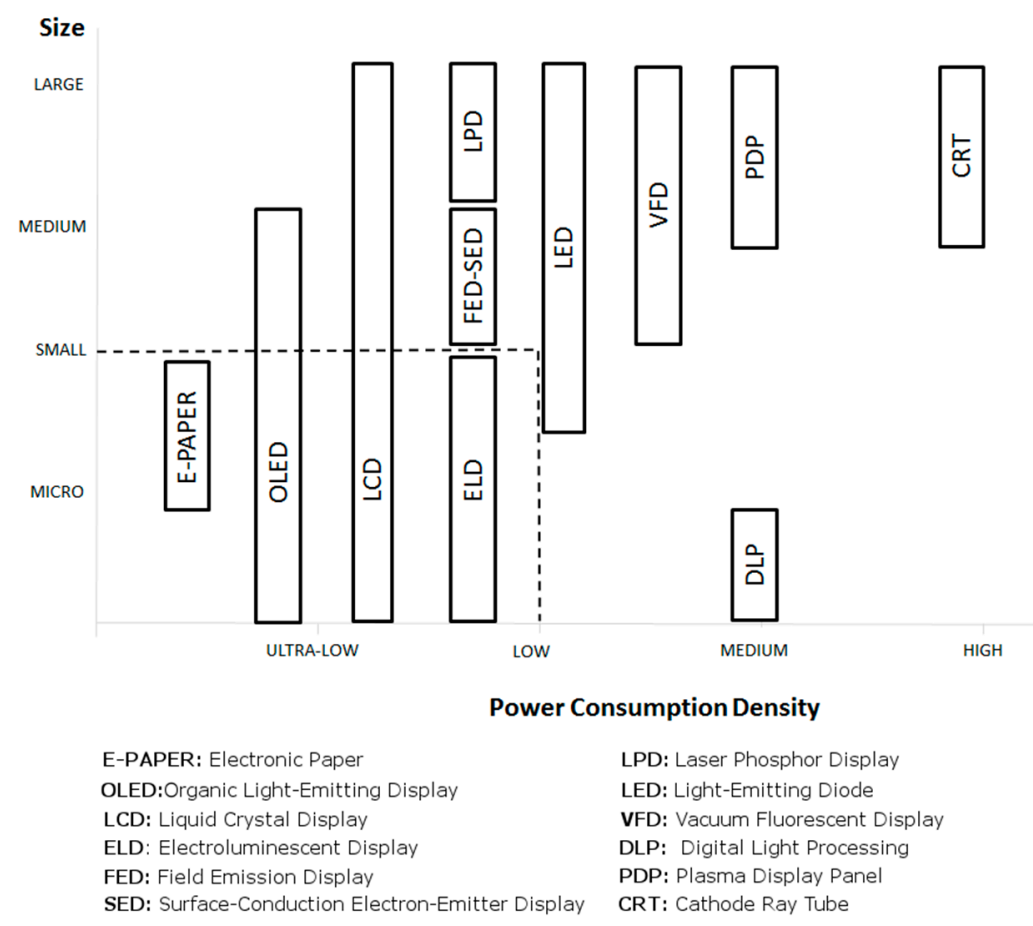

Figure 1. Relation between size and power consumption density in the studied display technologies. 


\section{Characterization of Displays}

According to the technology family, a display can be non-emissive, with a backlight in its design, or emissive, if it acts as a light source itself. Regarding the calculation of the power consumption, in the non-emissive family, the backlight is necessary for its full operation, so the power consumed by it must be included in the total power consumption (Pdisplay), as shown in Equation (1):

$$
P_{\text {display }}=P_{\text {panel }}+P_{\text {backlight }}
$$

Emissive displays show a remarkable increase in power consumption with size, while in reflective, the value remained almost proportional. Furthermore, the power consumption could be influenced by other features, such as human factors or technical specifications. The following display characteristics were taken into consideration in this study:

- The brightness, which is defined as the level of light intensity perceived by the viewer. It is estimated by the luminance or the amount of light emitted from a source in a given direction and it is measured in candela per square meter or nits. The contrast is also considered, determined as the ratio $(\mathrm{CR})$ between the luminance of the brightness and the darkest color that the display is capable of producing.

- The information content of a display, which is established as the total number of pixels, the size of them (resolution) and the size of the display (typically given by the diagonal length in inches). It is also common to provide the aspect ratio-proportion between the width and the height of the screen - and the screen area (normally measured in square centimeters).

- Other characteristics that can be influential in the consumption are the number of colors that the display can show and the angle of view (VA) - formally defined as the angle at which the viewer has to be positioned in relation to the screen in order to clearly see the image on a display.

\section{Low and Ultra-Low Power Consumption Microdisplay Technologies}

This section is organized regarding the specific technology used by the display subjects under study. For each technology, all the studied modules were grouped in categories with minimal dispersion of power consumption density. Within each group, the element with values closest to the mean, was selected as the most representative. For those selected modules, information about diagonal size (inches), weight $(\mathrm{g})$, brightness $\left(\mathrm{cd} / \mathrm{m}^{2}\right)$, contrast ratio (CR), degrees of viewing angle (VA) and absolute power consumption $(\mathrm{mW})$, obtained from the manufacturer is given in this section.

\subsection{Liquid Crystal Display (LCD)}

The term liquid crystal is used to describe a substance which is in a state between liquid and solid but exhibits the properties of both. The first observations of liquid crystalline behavior were made towards the end of the 19th century by Reinitzer and Lehmann [24], and since then, LCD technology has enjoyed significant advances and it currently occupies the largest proportion of the entire display market share [25]. 
LCD belongs to a non-emissive display category and can be classified in two broad categories, Passive Matrix (PMLCD) and Active Matrix (AMLCD). The basic difference between the two categories is the way the pixel is addressed to produce the different luminance components of an image.

Generally, the power consumption of any LCD is related to the drive frequency (the lower the frame rate becomes, the less power consumed) and the displayed image.

In PMLCD, pixels are addressed directly and they must retain their state between screen refreshes without the benefit of a steady electrical charge. It is used in devices where less content of information needs to be displayed and when the power consumption has to be reduced, since less backlight is needed.

In AMLCD, a switch is placed at each pixel which decouples the pixel-selection function. Thin Film Transistor (TFT), the main technology of the AMLCD subgroup, can also be divided regarding the material used for its elaboration, into amorphous silicon (a-Si), continuous grain silicon (CGS) and low temperature polycrystalline silicon (LTPS TFT). A new approach is the indium-gallium-zincoxide (IGZO) technology [26] developed by Sharp.

In addition, cholesteric LCD displays (ChLCD) is a novel LCD technology that only consumes power if the image is updated [27]. Due to its particular characteristics, this type of display will be studied in the e-paper section.

Table 2 provides technical data about the six selected LCD displays. The first row represents the PMLCD category, which having a power consumption close to average, offers a monochrome graphic display, in contrast to the AMLCD modules. The greater the number of pixels on a screen, the better the quality of the image produced, but as the number of pixels (and, correspondingly, columns and rows) increases, this type of display becomes less feasible, since under those conditions, it shows slow response times and poor contrast outcomes. Another issue to take into account is the liquid crystal alignment mode, where Twisted Nematic (TN) and Super-Twisted Nematic (STN) types are the simplest and least expensive, but offering a poor viewing angle (of approx. 45 degrees). Vertical Alignment (VA) technology generally appears under various trade names (ASV by Sharp, PVA by Samsung, etc.) and tries to improve the viewing angle of the device (for instance, Ampire VA device offers 160 degrees versus the 45 of the TN device by AUO). In-plane switching (IPS TFT), as the Hitachi module from the table shows, also has a better viewing angle than $\mathrm{TN}$ and the color and contrast is also improved. However, the power consumption required to make the molecules switch is higher.

Table 2. Features of specific Liquid Crystal Displays.

\begin{tabular}{cccccccc}
\hline Type & \multirow{2}{*}{ Model } & $\begin{array}{c}\text { Diag. } \\
\text { Size }(\boldsymbol{i n})\end{array}$ & $\begin{array}{c}\text { Weight } \\
(\boldsymbol{g})\end{array}$ & $\begin{array}{c}\text { Brightness } \\
\left(\mathbf{c d} / \boldsymbol{m}^{2}\right)\end{array}$ & $\boldsymbol{C R}$ & $\boldsymbol{V A}$ & $\begin{array}{c}\text { Power } \\
(\boldsymbol{m} \boldsymbol{W})\end{array}$ \\
\hline PMLCD (STN) & F-55471GNFJ [28] & 5.2 & 75.1 & 72 & $5: 1$ & 90 & 615 \\
a-Si TFT (TN) & A015AN04 [29] & 1.5 & 6 & 170 & $150: 1$ & 80 & 197 \\
a-Si TFT (VA) & AM-240320LFTZQW-00H [30] & 2.4 & TBD & 400 & $450: 1$ & 160 & 272 \\
a-Si TFT (IPS) & TX15D02VM0CAA [31] & 5.8 & 175 & 450 & $800: 1$ & 170 & 4900 \\
CG-S TFT & LS037V7DD06 [32] & 3.7 & 39 & 100 & $100: 1$ & 80 & 490 \\
LTPS TFT & ANDpSi025TH [33,34] & 2.5 & 15 & 250 & $300: 1$ & 80 & 216 \\
\hline
\end{tabular}


LCDs are used in a wide range of applications including televisions, monitors, instrument panels, video players, gaming devices, clocks, calculators or phones. For instance, LTPS LCD (Retina HD display) technology is the one used in the iPhone 6. In Figure 2, an example of this type of module can be seen.

(a)

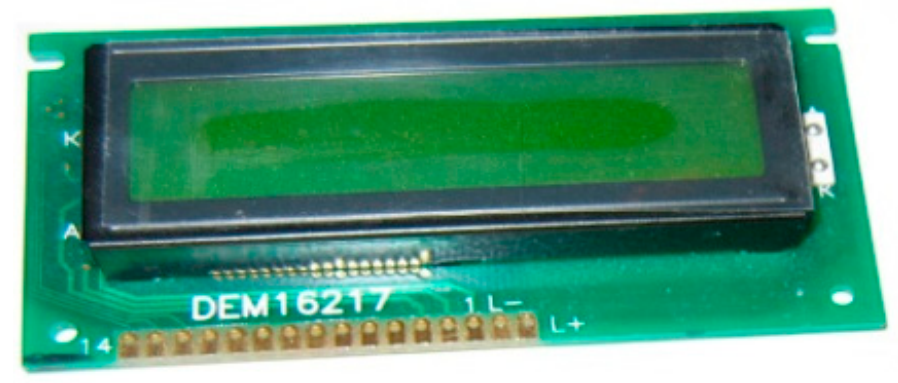

(b)

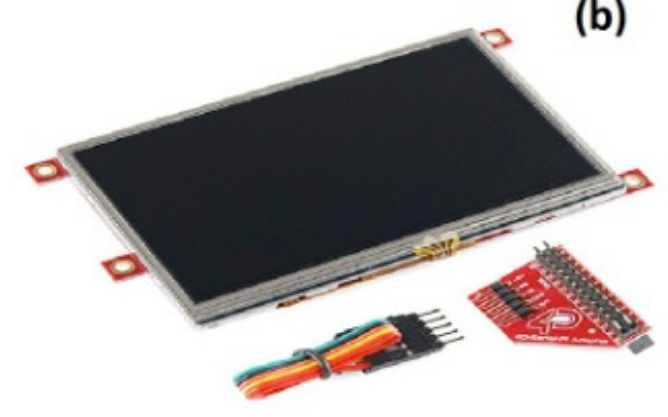

Figure 2. (a) Passive and (b) active matrix LCD modules (by SparkFun Electronics).

\subsection{Electronic Paper (E-Paper)}

Electronic paper, popularly known as e-paper, can be defined as a dynamic display technology that emulates traditional paper. As LCD, e-paper belongs to the non-emissive display category but, in this case, no backlight is needed since the ambient light from the environment is enough.

The display is composed of millions of microcapsules containing positively charged white and negatively charged black particles suspended in a clear liquid, which are capable of producing the resolution only found in print. As they are bi-stable, they only consume power while the display is being updated. The power required for the update process depends on the size of the display.

The first commercial success of monochrome e-paper devices was due to the Electrophoretics technology, wrongly referred to as electronic paper displays (EPD), whose main exponent is microencapsulated electrophoretic displays, also known as e-ink [35]. Another similar approach, microcellular electrophoretic display films (SiPix), was bought by e-ink. There are other proprietary electrophoretic displays, which include Quick-Response Liquid Powder Display (QR-LPD) by Bridgestone [36], bichromal beads [37] by Xerox (Gyricon), or reverse emulsion electrophoretic display (REED) used by Zikon Corp.

Cholesteric liquid crystal (ChLCD), already mentioned as a subgroup of LCD, is generally classified as e-paper because of its zero consumption when it is not receiving screen updates. Figure 3a, below, shows a cholesteric display powered by solar panels.

A next generation of flexible, color and video e-paper is currently emerging. The most promising seems to be the electro-wetting approach (EWD) [38]. Its main component, liquavista (see Figure 3b), was developed by Philips but currently belongs to Amazon. Another interesting technology based on Interferometric Modulation (IMOD) is microelectromechanical sytems (MEMS) [39], whose potential has been demonstrated through several prototypes (trademarked Mirasol [40]) developed by Qualcomm. 


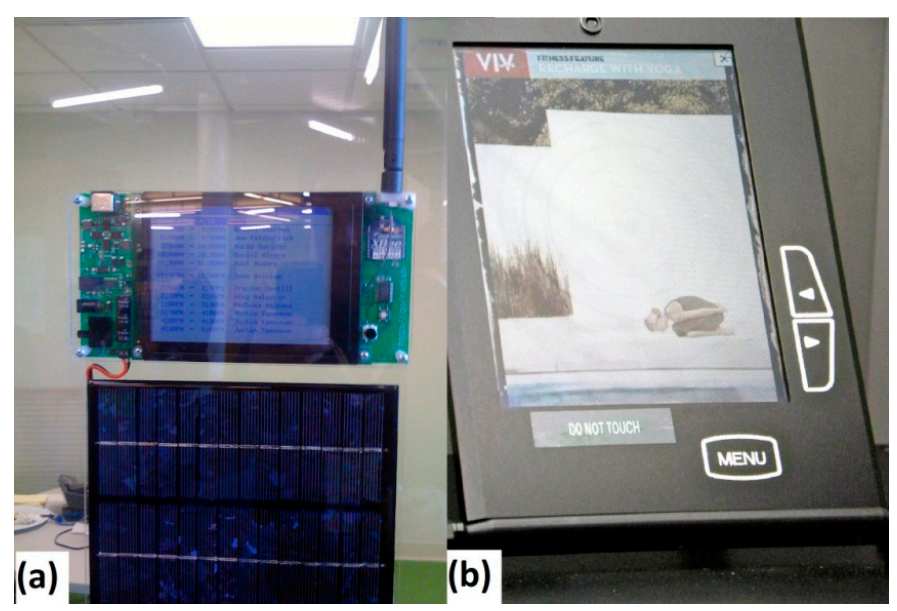

Figure 3. (a) Google Radish project using solar panels and a cholesteric display (by Niall Kennedy) and (b) Liquavista prototype.

Some other remarkable developments are the in-plane electrophoretics (IPE) patented by Canon [41] and HP's Electrokinetic (EKD) [42], although they are at least several years away from a general market uptake [43].

Another less matured technology is electrofluidic [44], which presents the main novelty of using a three-dimensional microfluidic device structure and offering brilliantly colored aqueous pigment dispersions. Recently, other lines of investigation have aimed to simulate traditional paper through electronic paper made from microbial cellulose [45].

Generally, the main characteristics of electronic paper are flexibility, readability, multi-functionality and ultra-low power consumption-zero power consumption during the non-updating period [46]. It is common to find this kind of technology in small sizes, although there are approaches with A5 size [47]. The majority are monochrome but there are also color options such as e-ink [48] or electrochromic (EC) technologies [49]. Liquavista, the recent acquisition of Amazon, also allows colors to be incorporated into the display. In addition, the best contribution of liquavista is that its low consumption remains almost constant in all modes of operation, while e-ink offers good levels for e-books, but rises a lot in operations requiring interaction with the user, such as web browsing.

Table 3, below, lists e-paper displays with their technical data.

Table 3. Features of specific electronic paper displays.

\begin{tabular}{ccccccc}
\hline Type & Model & Diag. Size (in) & Weight (g) & CR & VA & Power (mW) \\
\hline EPD & GDE021A1 [50] & 2.1 & 4 & $7: 1$ & 180 & 24 \\
Pearl EPD & ED060SCE [51] & 6 & 34 & $12: 1$ & 180 & 240 \\
ChLCD & LCD-09559 [52] & 5.5 & 105 & $25: 1$ & 180 & 150 \\
EWD & Liquavista prototype (Amazon) [53] & 2.5 & TBD & $18: 1$ & 180 & 87 \\
\hline
\end{tabular}

The most common applications of e-paper are eReaders, eLabels, USB sticks, clocks, billboards, etc. A good commercial example is the Kindle by Amazon that uses e-ink technology. 


\subsection{Organic Light-Emitting Display (OLED)}

The Organic Light-Emitting Diode (OLED) is a light-emitting technology made by placing a series of thin organic films (made from carbon and hydrogen) between two conductors. When electrical current is applied, a bright light is emitted.

There are several development trends in the field of organic diodes and the most representative classification, based on the driving circuitry, is the active matrix OLED (AMOLED) [54-56] and the passive matrix OLED (PMOLED) [57].

Depending on the light type, different types can be distinguished, such as phosphorescent Organic Light-Emitting Diode (PhOLED), Transparent Organic Light-Emitting Diode (TOLED), or White Organic Light-Emitting Diode (WOLED).

Because of its nature, it is possible to create flexible and transparent interfaces [58]. When an OLED screen is marked with a flexible plastic substrate, such as a Polymer Light-Emitting Diode (PLED), it is called a Flexible Organic Light-Emitting Diode (FOLED). Other OLED advances have been achieved by using molecules in its composition, but they are still in the development process; some examples might be the Molecule Organic Light-Emitting Diode (MOLED) [59] and the Small Molecule Organic Light-Emitting Diode (SmOLED) [60].

All the OLED displays have some characteristics in common: high brightness and contrast, fast response time and excellent color definition. They offer a wide viewing angle (at around 160 degrees) as a result of the self-luminous effect. The main advantage, however, is their low power consumption (proportional to the number of pixels that are turned on-black dots do not need power), which depends only on the present content due to the fact that they do not require a backlight. This also makes them thinner and more efficient [61]. The handicap is still the price; since they are manufactured on a small scale, they have a high price on the market [60].

Some studies [62] have concluded that when using this technology, the power consumption of displays increases very strongly with size. Displays of different sizes within the studied range have therefore been analyzed and their features are listed in Table 4. Such increase is especially remarkable in the case of PMOLED (a module twice as large consumes six times more).

Table 4. Features of specific Organic Light-Emitting Displays.

\begin{tabular}{cccccccc}
\hline Type & Model & $\begin{array}{c}\text { Diag. } \\
\text { Size (in) }\end{array}$ & $\begin{array}{c}\text { Weight } \\
(\boldsymbol{g})\end{array}$ & $\begin{array}{c}\text { Brightness } \\
\left(\mathbf{c d} / \boldsymbol{m}^{2}\right)\end{array}$ & $\boldsymbol{C R}$ & $\begin{array}{c}\text { VA A } \\
(\boldsymbol{m} \boldsymbol{W})\end{array}$ \\
\hline AM-OLED & C0201QILK-C [63] & 2 & 6 & 190 & $10000: 1$ & 170 & 170 \\
AM-OLED & USMP-A34480TP (Chi Mei El. Corp) [64] & 3.4 & 30.1 & 160 & $10000: 1$ & 170 & 500 \\
PM-OLED & microOLED-160-G2 [65] & 1.7 & 13 & 100 & $5000: 1$ & 160 & 100 \\
PM-OLED & RGS32256064WH002 [66] & 3.2 & 11 & 70 & $2000: 1$ & 160 & 616 \\
\hline
\end{tabular}

OLED technology is being conducted in the main companies and universities, such as Kodak, Sharp or eMagin. Although it started being used mainly in cameras, A/V players, car audio systems or other small devices such as the smart watch shown in Figure 4, the market share in mobile screens is increasing. For instance, Super AMOLED is the technology chosen by Samsung for its Galaxy S6. 


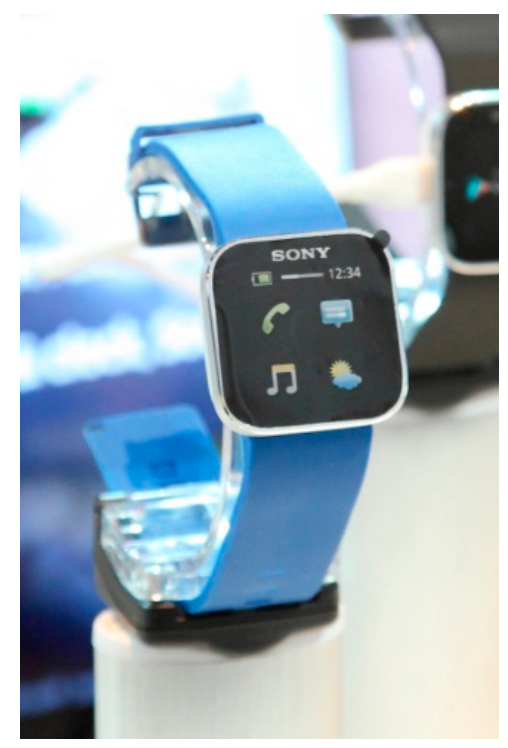

Figure 4. Sony SmartWatch with an OLED display (by Bim imGarten).

\subsection{Electroluminescent Display (ELD)}

Electroluminescent Displays (ELD) have their origins in the first decade of the twentieth century, but they did not become commercially viable products until the 1980s.

Electroluminescent display technology takes advantage of the light-emission phenomenon due to a strong electric field. It consists of a solid state thin phosphor film and insulator stack deposited on a glass substrate and driven by high voltage electronics which generate alternating positive and negative pulses [67]. This is a very cost efficient light source, which results in low power consumption.

ELD displays belong to the emissive category and besides, they can be classified into two main groups. The first, thick-film dielectric electroluminescent (TDEL), is a phosphor-based flat panel display technology. TDEL is based on inorganic electroluminescent (IEL) technology and has a novel structure that combines both thick and thin-film processes. In the case that it incorporates a black thick film dielectric layer, it is called black thick-dielectric EL (BDEL). Thin-film electroluminescent (TFEL) [68] consists of a self-healing metal row electrode, two dielectrics sandwiching a light-emitting phosphor, which emits light via hot-electron impact excitation of luminescent dopants, and a transparent indium tin oxide (ITO) column electrode.

Color ELD technology has advanced significantly in recent years, especially for microdisplays. Other remarkable research still in progress in the electroluminescent field includes the Active Matrix Electroluminescence (AMEL) and the Transparent Electroluminescent Displays (TASEL) [69], such as the one shown in Figure 5.

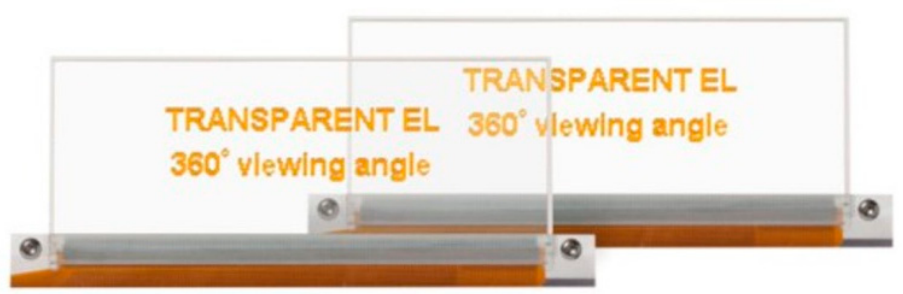

Figure 5. Fully transparent TFEL Display (by Beneq Oy, Finland). 
Electroluminescent technology is characterized by having low power consumption and low contrast ratios. Attention should also be paid to the viewing angle values which are around 180 degrees, achieving 360 in the case of transparent TASEL displays [70]. Table 5 contains the studied feature values for four representative ELD displays. In the case of TDEL and AMEL, the values for a prototype are given since they have not yet been commercialized. The two main firms that have developed and commercialized this technology are Sharp and Planar Systems.

Table 5. Features of specific Electroluminescent Displays.

\begin{tabular}{cccccccc}
\hline \multirow{2}{*}{ Type } & Model & $\begin{array}{c}\text { Diag. } \\
\text { Size (in) }\end{array}$ & $\begin{array}{c}\text { Weight } \\
(\boldsymbol{g})\end{array}$ & $\begin{array}{c}\text { Brightness } \\
\left(\mathbf{c d} / \boldsymbol{m}^{2}\right)\end{array}$ & $\boldsymbol{C R}$ & $\begin{array}{c}\text { VA } \\
\text { Power } \\
(\boldsymbol{m} \boldsymbol{W})\end{array}$ \\
\hline AMEL & VGA prototype (Planar) [67] & 0.758 & 2.1 & 342 & $100: 1$ & 160 & 400 \\
TDEL & TDEL prototype (TDK Corp) [71] & 4.25 & 284 & 200 & $3: 1$ & 180 & 10,000 \\
TFEL & EL320.240.36 HB [72] & 5.7 & 180 & 150 & $90: 1$ & 160 & 3500 \\
TFEL & LJ32H028 [73] & 4.7 & 270 & 200 & $300: 1$ & 160 & 5000 \\
\hline
\end{tabular}

\section{Results and Discussion}

In order to facilitate the drawing of conclusions from the technical data presented in the previous section, the main features are graphically shown in Figure 6. The radar charts allow comparing the relative weight, brightness, viewing angle and contrast ratio values between the different display technology groups. For those categories in which more than one element has been studied, the mentioned features presented uniform outcomes; hence the average value was taken as a reference to make comparisons with other technologies.

To compare weights, the relative value (weight to active area) was calculated. All technologies offer a value close to $1 \mathrm{~g} / \mathrm{cm}^{2}$, with the exception of TFEL that stands with mean values of $2.9 \mathrm{~g} / \mathrm{cm}^{2}$, reaching highs of up to $4 \mathrm{~g} / \mathrm{cm}^{2}$.

Two technologies are presented at the ends when the brightness values are analyzed: on one side, e-paper where, lacking light emission, the brightness can be considered zero; and second, AMLCD, where the average brightness of some modules reaches values of $450 \mathrm{~cd} / \mathrm{m}^{2}$.

Regarding the viewing angle, LCD is the technology that provides the worst average results, but that can be improved by using new methods of alignment, as is the case of VA or IPS, which allows viewing angles up to 170 degrees. In this aspect, EL displays highlights with values from 160 up to 360 degrees in the innovative TASEL transparent display.

Finally, the contrast ratio is considerably lower in PMLCD and e-paper, but enough for the kind of applications that these technologies are designed for.

Focusing on the characteristics concerning power consumption, Table 6 shows a comparison of the main parameters for each of the studied display technologies. 


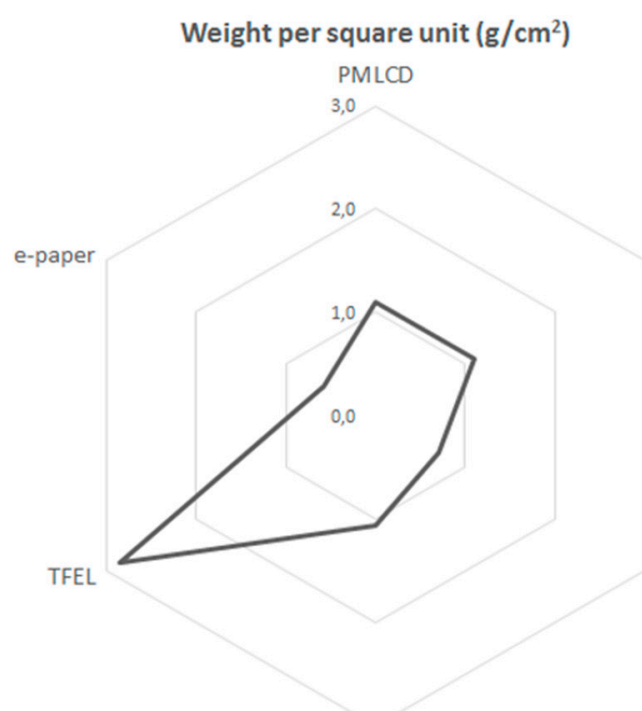

PMOLED

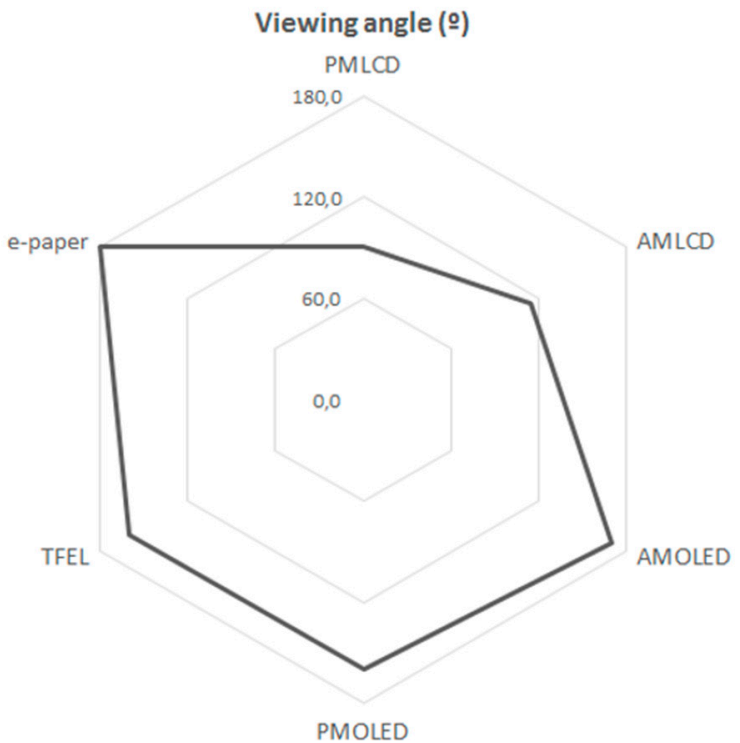

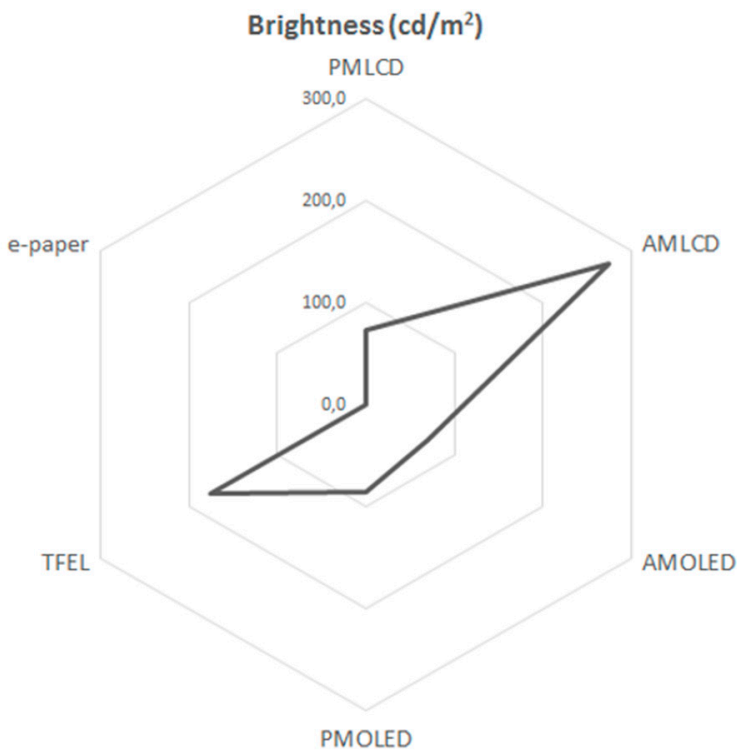

Contrast ratio

PMLCD

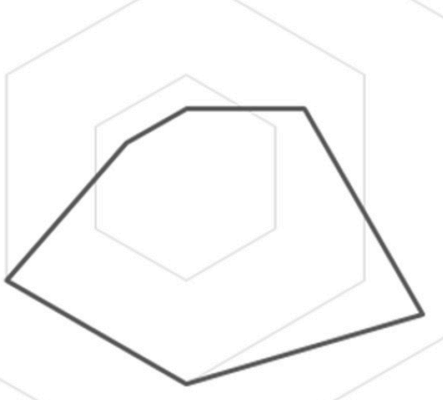

AMLCD

-paper

PMOLED

Figure 6. Radar charts of relative weight, brightness, viewing angle and contrast of studied technologies.

Table 6. Power consumption-related features comparison.

\begin{tabular}{|c|c|c|c|c|c|c|}
\hline & Parameter & STN-LCD & TFT-LCD & EPD & OLED & ELD \\
\hline \multirow{5}{*}{ 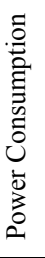 } & Typ. $(\mathrm{mW})^{*}$ & $100-200$ & $200-300$ & $25-50$ & $150-200$ & $1500-2000$ \\
\hline & Max. $(\mathrm{mW}) *$ & 1000 & 300 & 100 & 1000 & 5000 \\
\hline & Proportional to size & NO & NO & YES & YES & YES \\
\hline & Stable state & YES & YES & NO & YES & YES \\
\hline & Black image & YES & YES & NO & NO & NO \\
\hline & Backlight & YES & YES & NO & NO & NO \\
\hline & wer saving mode & NO & YES & NO & YES & YES \\
\hline & Unit cost & Low & Medium & Medium/high & Medium/high & High \\
\hline & Iain applications & Cheap electronics, toys & Wide range applications & E-book, watches & Mobile & Embedded devices \\
\hline & Weak points & Resolution & Visual quality & Color, response time & Lifespan & Size, consumption \\
\hline
\end{tabular}

* Range values for 1.5-2.5 inches displays. 
The results exposed until now, offer a first characterization of each type of display, but relative measures related with the power consumption are needed to make accurate and reliable comparisons between displays of different natures.

The amount of power per unit area or surface power density $\mathrm{P}_{\mathrm{d}}$ (measured in $\mathrm{mW}$ per square centimeter of active screen area) can be used (see Equation (2)).

$$
P_{d}=\frac{P}{S}
$$

A comparison of the power density of the studied displays is shown in Figure 7.

As the chart stands out, on the one hand, electronic paper modules (EPD, EWD and ChLCD) offer the lowest power consumption values, but it is necessary to take into account the fact that this technology is used for the development of very specific displays (typically monochrome, focused to display text, allowing sunlight readability, etc.). The shortcoming is that e-papers are not viewable without ambient light. Additionally and more importantly, it uses little to no power to preserve a static image on the screen.

Close to the same power density value but even higher is LCD technology. The passive matrix PMLCD and the active matrix, a-Si TFT (with Vertical Alignment mode) obtain the best results, although there is a subtype of LCD with high consumption, a- Si TFT IPS, because of the high power required to make the molecules switch.

Finally, within the range defined as low power consumption, electroluminescent devices are the most power-consuming ones.

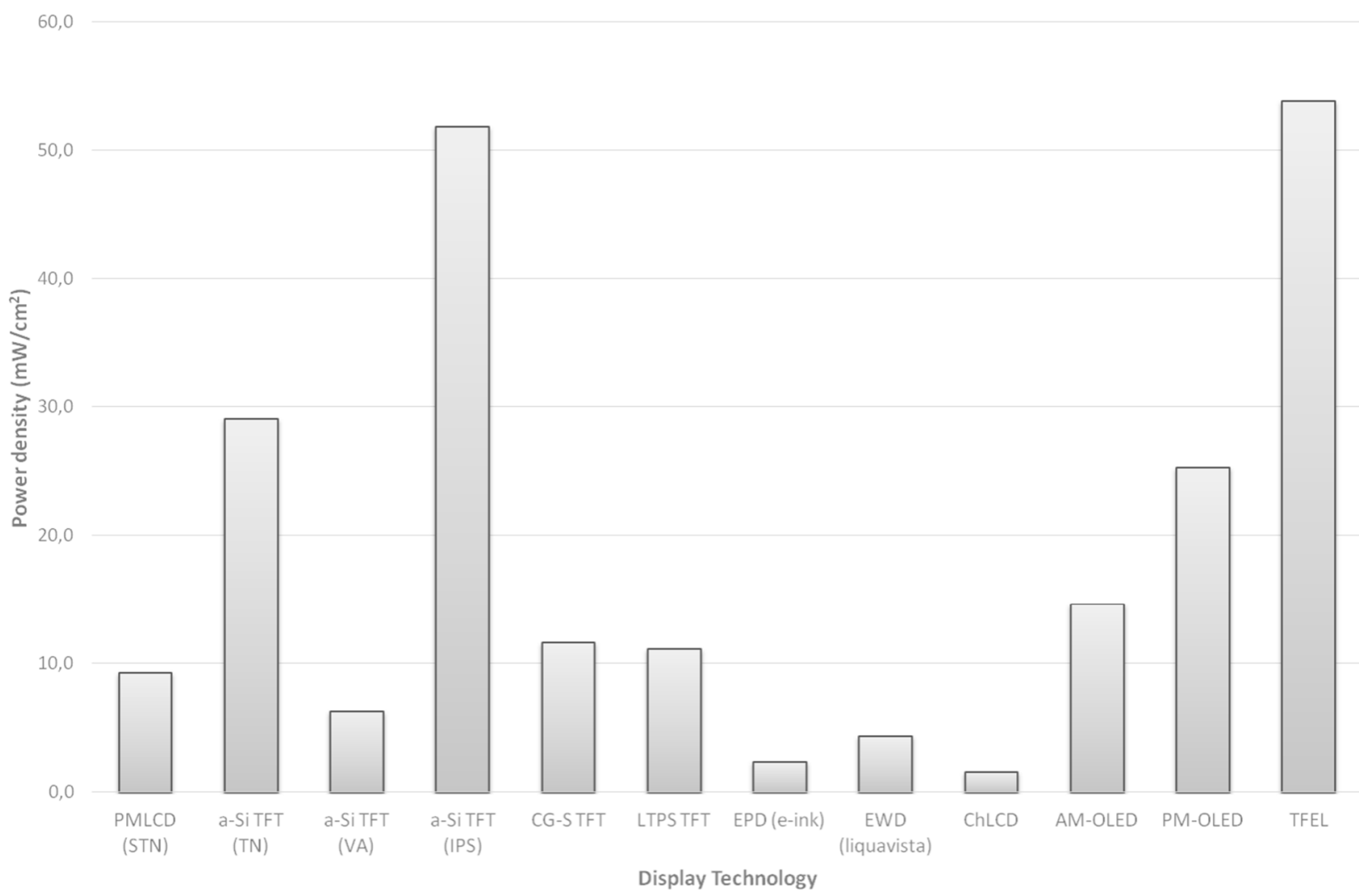

Figure 7. Power density of studied display technologies. 
Due to the influence of what is being displayed on the screen in the consumption of some displays, it seems appropriate to perform a comparison while keeping constant that pattern. The display intensity efficiency or the energy efficiency of a display has been used. It is defined by the IDMS as the ratio of the luminous intensity I (defined as the product of the frontal luminance $\mathrm{L}_{\mathrm{W}}$ of the white full screen and the active area S of the display, as is shown in Equation (3)) to the power consumption P (see Equation (4)). It is measured in candle per watt.

$$
\begin{gathered}
I=L_{W} S \\
\xi=\frac{\mathrm{I}}{\mathrm{P}}
\end{gathered}
$$

After analyzing the energy efficiency values for the studied modules (electronic paper displays have not being taken into account because the frontal luminance efficiency is not valuable as a figure of merit), significant differences depending on whether the type of display is emissive or non-emissive have been detected. A comparison between LCD and OLED modules (differencing between active and passive technologies) is shown in Figure 8. Although outside the scope of the study, for larger sizes, OLED will perform worse than LCD in terms of energy efficiency.

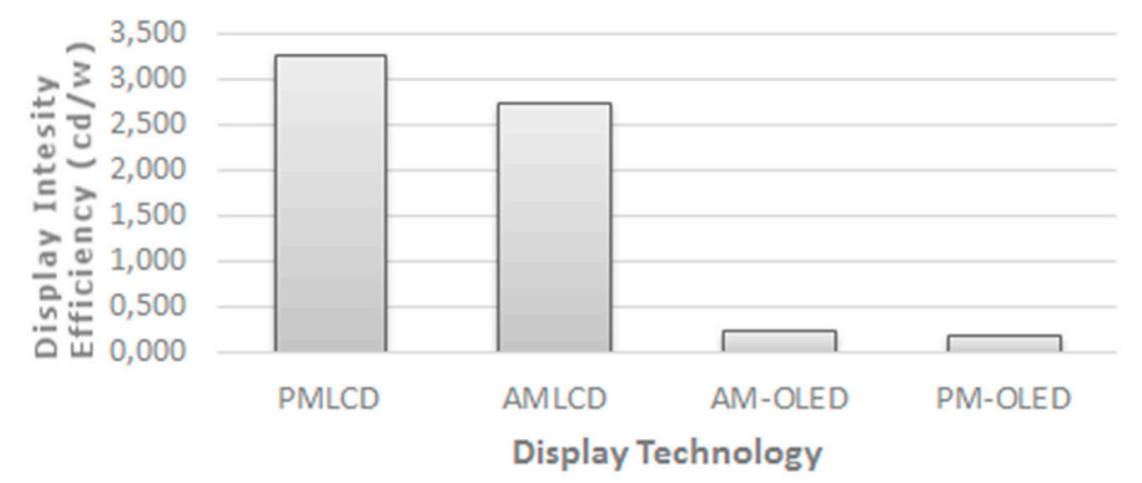

Figure 8. Comparison of display intensity efficiency of LCD and OLED in their active and passive versions.

\section{Applications and Trends}

Novelty lines of research are exploring new fields of application. Since there are no commercial devices until the technology reaches a mature stage, an accurate idea of the current trends can be obtained through an analysis of the patent networks as proposed by Chang [74].

Microdisplays are the basis that enables projection displays to screen large images from small dimension devices, thereby offering lower power consumption rates at reduced cost and under extremely portable conditions. An extension would be near-to-eye (NTE) systems that require the user to place the display close to their eyes with a head-mounted display, offering the same mentioned advantages. An emerging field of study of this see-through display is the occlusion capability, which enhances user's perception, visibility and realism [75].

Another new trend of research during the last few years has been focused on developing flexible displays in curved, conformal, bendable and even rollable shapes. This opens up the possibility of incorporating new applications and products into the market. The key of this approach is the material 
employed. The use of polymers [76], as well as graphene [77], seems very promising in terms of consumption. There are prototypes of different technologies and sizes, from a large plasma display [78] to a small AMOLED module [79] passing through the Mobius Display by E-ink [80], which maintains the power consumption levels of other e-ink displays.

$3 \mathrm{D}$ displays are one of the most futuristic approaches but they are already a reality. The first attempts used 2D technologies, such as LCD or DLP [81], and a special device, commonly glasses, for offering the stereoscopic effect (a different picture to each eye). One step further, the autostereoscopic technology [82] is based on the previous and displays multiple different images on one display screen, each visible from particular places in front of the screen [83]. A widespread commercial device is Nintendo 3DS.

Besides the evolution of the technology, another complementary field of action to reduce the display consumption waste is the improvement in user interaction and usability of the interface itself [84]. Other research includes working on the adaptation of the software to conserve energy [85]. Display power management (DPM) policies can reduce the energy used for the display integrated in a system turning off and on the display depending on the attention of the user. As this action can result in unacceptable quality degradation, the need of new energy reduction and optimization techniques arises [86].

The main ones - LCD displays - are covered in the survey of Anggorosesar [87], which classifies the low-power techniques in four categories: backlight dimming, dynamic voltage scaling, software-based and hardware-based. They can achieve power saving ratios by up to $90 \%$ of the total system power with a small distortion level. Each of the techniques saves the power consumption of the display system by reducing the activity of the corresponding components such as the color depth [88], the refresh duty ratio [89], the frame buffer [90] or the backlight luminance. In the last approach, dynamic luminance scaling (DLS) of the backlight, with appropriate image compensation, stands out. Its extended version, EDLS, compensates for loss of brightness when the number of saturated pixels is small, and for loss of contrast when there are too many saturated pixels in the image [91].

Regarding emissive displays, especially OLED, although the previously described techniques can also be applied, there are more specific studies based on the idea that the relationship between intensity levels of color components and power consumption is not linear. For example, Dong et al. present three different models to provide power estimation for the system to manage and optimize energy consumption [92]. For its part, Chen et al., use dynamic voltage scaling (DVS) for the power management of the display on mobile devices in video streaming applications [93]. Other approaches propose using a more efficient code such as RGBW, obtaining less color distortion with less power consumed [94].

The possibility of combining the advantages of each technique into one collaboration to improve the whole power system is research for the future [87].

\section{Conclusions and Future Directions}

The small-sized low-power displays are usually geared in the household context to phones, multimedia devices, navigators, etc.; while in the professional world, the automotive sector and industrial applications and appliances are the main users. Going beyond, in energy harvesting 
environments [95], where devices present extreme low-power consumption needs, high energy efficient displays could play an essential role.

Although great strides have already been made to reduce the consumption of the interfaces, it is still a main objective of industrial strategic agendas and research groups' lines of investigation.

This review has compiled relevant data related with the energy consumption of the main low-power display technologies, from the manufacturers' datasheets. The use of relative units has allowed comparing them accurately.

Some display technologies, such as OLED—reflective display with no backlight—or EPD—which retains the shown information-have intrinsically low power characteristics in small form factors. Several market reports place also OLED as a key technology up to 2020 [96]. It has the best contrast ratios and viewing angles, and good values for brightness and weight. The strong relationship between the number of active pixels and the power consumed makes it suitable for many specific applications.

Electronic paper is also a promising technology for applications that need ultra-low-power displays. Especially for those that require infrequent updating of images, this display consumes an extremely low amount of power.

Other more mature technologies are also concentrating on low-power small-sized display development, such as LCD. Even though it is making progress, it must continue to improve if it wants to remain competitive against other emerging display technologies.

Although ELD displays perform poorly in terms of energy, they present usage advantages under circumstances where full color is not required but where ruggedness, speed, brightness, high contrast, and a wide angle of vision is needed.

Display technologies must evolve fast enough to keep pace with advances in other areas. Each day new ways are devised with the aim of improving the brightness, contrast, and overall picture quality of the displays, but the demand for low energy-consuming displays is one of the main milestones that will drive technology evolution; this evolution will require new approaches and innovative ideas. New lines of research are thus exploring new fields of application to meet the changing needs of society.

\section{Acknowledgments}

This research has been partially supported by the Junta de Castilla y León-Agencia de Inversiones y Servicios y Programa de Apoyo a Proyectos de Investigación (grant VA036U14) and Ministry of Science and Innovation (grant DPI2014-56500-R).

The authors would also like to thank University of Oviedo for hosting this research and especially the staff of the Infobotica Research Group for all the valuable comments.

\section{Author Contributions}

All authors contributed extensively to the work presented in this paper. Ignacio Gonzalez Alonso and Eduardo Zalama Casanova designed and structured the contents. María Rodríguez Fernández gathered the information, analyzed the data and wrote the paper. All authors discussed the results and implications and commented on the manuscript at all stages. 


\section{Conflicts of Interest}

The authors declare no conflict of interest.

\section{References and Notes}

1. Cranston, G.R.; Hammond, G.P. Egalite, fraternite, sustainabilite: Evaluating the significance of regional affluence and population growth on carbon emissions. Int. J. Glob. Warm. 2010, 2, 189-210.

2. Fehske, A.; Fettweis, G.; Malmodin, J.; Biczok, G. The global footprint of mobile communications: The ecological and economic perspective. IEEE Commun. Mag. 2011, 49, 55-62.

3. Gielen, D. Energy Technology Perspectives; Paris International Energy Agency: Paris, France, 2008.

4. Carroll, A.; Heiser, G. An Analysis of Power Consumption in a Smartphone. In Proceedings of the 2010 USENIX Conference on USENIX Annual Technical Conference, Boston, MA, USA, 23-25 June 2010; p. 21.

5. Pitt, M.G.; Zehner, R.W.; Amudson, K.R.; Gates, H. 53.2: Power Consumption of Micro-Encapsulated Display for Smart Handheld Applications; SID Symposium Digest Technical Papers; Wiley: Hoboken, NJ, USA, 2002; Volume 33, pp. 1378-1381.

6. Simunic, T.; Benini, L.; Glynn, P.; De Micheli, G. Event-driven power management. Comput. Aided. Des. Integr. Circuits Syst. IEEE Trans. 2001, 20, 840-857.

7. Kimmel, J. Energy Aspects of Mobile Display Technology. In Handbook of Visual Display Technology; Springer: Berlin, Germany, 2012; pp. 2023-2030.

8. Myers, R.L.; Wiley, J. Display Interfaces: Fundamentals and Standards; Wiley: Hoboken, NJ, USA, 2002.

9. International Committee for Display Metrology Information Display Metrology Standard Website. Available online: http://icdm-sid.org/ (accessed on 31 July 2015).

10. SID, The Society for Information Display Website. Available online: http://www.sid.org/ (accessed on 31 July 2015).

11. Mendicino, L. Environmental Issues with Materials and Processes for the Electronics and Semiconductor Industries: Proceedings of the Fourth International Symposium; The Electrochemical Society: Pennington, NJ, USA, 2001.

12. Specification for the assessment of the life cycle greenhouse gas emissions of goods and services. Available online: http://shop.bsigroup.com/upload/Shop/Download/PAS/PAS2050.pdf (accessed on 8 August 2015).

13. Smil, V. Energy in Nature and Society: General Energetics of Complex Systems; MIT Press: Cambridge, MA, USA, 2008.

14. Japan Display Inc. Available online: http://www.j-display.com/english/ (accessed 31 July 2015).

15. The German Flat Panel Display Forum. Available online: http://www.displayforum.de/ (accessed on 31 July 2015).

16. Grand View Research Inc. Microdisplays Market Analysis, Market Size, Application Analysis, Regional Outlook, Competitive Strategies and Forecasts, 2015 To 2022. Available online: http://www.grandviewresearch.com/industry-analysis/microdisplays-market (accessed on 31 July 2015). 
17. Bray, M. Review of Computer Energy Consumption and Potential Savings; Dragon System Software Ltd. (DssW): Hereford, UK, 2006.

18. Talin, A.A.; Dean, K.A.; Jaskie, J.E. Field Emission Displays: A critical review. Solid-State Electron. 2001, 45, 963-976.

19. Kariyawasam, T. Field Emission of Carbon Nanotubes. Available online: http://www.phys.lsu.edu/ jarrell/COURSES/ELECTRODYNAMICS/Student_Projects/tharanga/r eview.pdf (accessed on 6 August 2015).

20. Komoda, T.; Koshida, N. Nanocrystalline Silicon Ballistic Electron Emitter. In Device Applications of Silicon Nanocrystals and Nanostructures; Springer: Berlin, Germany, 2009; pp. 251-291.

21. Yamamoto, K.; Nomura, I.; Yamazaki, K.; Uzawa, S.; Hatanaka, K. 71.2: Fabrication and Characterization of Surface Conduction Electron Emitters; SID Symposium Digest of Technical Papers; Wiley: Hoboken, NJ, USA, 2005; Volume 36, pp. 1933-1935.

22. Betsui, K. Advanced manufacturing technologies on color plasma displays. SPIE 2000, doi:10.1117/12.389427.

23. Armitage, D.; Underwood, I.; Wu, S.-T. Introduction to Microdisplays; Wiley: Hoboken, NJ, USA, 2006.

24. Reinitzer, F. Beiträge zur kenntniss des cholesterins. Monatshefte. Chem. Chem. Mon. 1888, 9, 421-441.

25. Kim, K.-H.; Song, J.-K. Technical evolution of Liquid Crystal Displays. NPG Asia Mater. 2009, 1, 29-36.

26. Lee, J.; Kim, D.; Yang, D.; Hong, S.; Yoon, K.; Hong, P.; Jeong, C.; Park, H.-S.; Kim, S.Y.; Lim, S.K. 42.2: World's Largest (15-Inch) XGA AMLCD Panel Using IGZO Oxide TFT; SID Symposium Digest of Technical Papers; Wiley: Hoboken, NJ, USA 2008; Volume 39, pp. 625-628.

27. Jones, J.C. The Zenithal Bistable Display: From concept to consumer. J. Soc. Inf. Disp. 2008, 16, $143-154$.

28. Kyocera F-55471GNFJ Display Specifications; KYOCERA Display Corporation.

29. AU Optronics General Display products Specifications. Available online: http://www.auo.com/ $? \mathrm{sn}=149 \&$ lang=en-US\&c=35 (accessed on 31 July 2015).

30. Ampire Specifications for LCD Module AM-240320LFTZQW-00H, 2011.

31. Kaohsiung Hitachi Electronics Co. Customer's Acceptance Specification TX15D02VM0CAA. Available online: http://static1.1.sqspcdn.com/static/f/489821/16638760/1389725608473/ TX15D02VM0CAA.pdf (accessed on 6 August 2015).

32. Sharp Corporation Device Specification for CG-Silicon TFT-LCD Module LS037V7DD06, 2004.

33. Nakajima, Y.; Teranishi, Y.; Kida, Y.; Maki, Y. 22.4: Invited Paper: Ultra-Low-Power LTPS TFT-LCD Technology Using a Multi-Bit Pixel Memory Circuit; SID Symposium Digest Technical Papers; Wiley: Hoboken, NJ, USA, 2006; Volume 37, pp. 1185-1188.

34. Japan Display Inc. Japan Display Introduces Paper-like Color Reflective LCD. Available online: http://www.j-display.com/english/news/2012/20121025.html (accessed on 31 July 2015).

35. Comiskey, B.; Albert, J.D.; Yoshizawa, H.; Jacobson, J. An electrophoretic ink for all-printed reflective electronic displays. Nature 1998, 394, 253-255. 
36. Hattori, R.; Masuda, Y.; Nihei, N.; Sakurai, R.; Yamada, S. Power consumption of a Quick-Response Liquid Powder Display (QR-LPD). IMID 2005, 5, 845-848.

37. Sheridon, N.K. Some Uses of Microencapsulation for Electric Paper. U.S. Patent, 5,604,027, 18 February 1997.

38. Hayes, R.A.; Feenstra, B.J. Video-speed electronic paper based on electrowetting. Nature 2003, 425, 383-385.

39. Miles, M.W. A new reflective FPD technology using interferometric modulation. J. Soc. Inf. Disp. 2012, 5, 379-382.

40. Qualcomm Mirasol Display Technology Website. Available online: http://www.qualcomm.com/ mirasol (accessed on 31 July 2015).

41. Liang, R.; Chung, J.; Chen, D. Electrophoretic Display with in-Plane Switching. U.S. Patent, 6,885,495 B2, 27 July 2005.

42. Zhou, Z.-L.; Liu, Q.; Yeo, J.-S.; Combs, G.; Benson, B.; Parent, M.; Yang, J.; Mabeck, J.; Lam, S.; Jeon, Y. Development of Novel Electronic Inks for Print-Like Color Reflective Display; Hewlett-Packard Development Company: Palo Alto, CA, USA, 2011.

43. Liu, B.B.Q.; Koch, T.R.; Mabeck, J.; Hoffman, R.L.; Mourey, D.A.; Combs, G.; Zhou, Z.-L.; Henze, D. 52.4L: Late-News Paper: Ultra-Low-Power Reflective Display with World's Best Color; SID Symposium Digest Technical Papers; Wiley: Hoboken, NJ, USA, 2012; Volume 43, pp. 708-710.

44. Yang, S.; Heikenfeld, J.; Kreit, E.; Hagedon, M.; Dean, K.; Zhou, K.; Smith, S.; Rudolph, J. Electrofluidic displays: Fundamental platforms and unique performance attributes. J. Soc. Inf. Disp. 2012, 19, 608-613.

45. Shah, J.; Malcolm Brown, R. Towards electronic paper displays made from microbial cellulose. Appl. Microbiol. Biotechnol. 2005, 66, 352-355.

46. Omodani, M. 10.1: Invited Paper: What is Electronic Paper? The Expectations. In SID Symposium Digest of Technical Papers; Wiley: Hoboken, NJ, USA, 2004; Volume 35, pp. 128-131.

47. Gates, H.; Zehner, R.; Doshi, H.; Au, J. 31.2: A5 Sized Electronic Paper Display for Document Viewing; SID Symposium Digest of Technical Papers; E-Link: Cambridge, MA, USA, 2012; Volume 36, pp. 1214-1217.

48. Hiji, N.; Machida, Y.; Yamamoto, Y.; Satoh, Y.; Ootani, S.; Satoh, T.; Shigemura, K. 8.4: Distinguished Paper: Novel Color Electrophoretic E-Paper Using Independently Movable Colored Particles; SID Symposium Digest Technical Papers; Wiley: Hoboken, NJ, USA, 2012; Volume 43, pp. 85-87.

49. Jeon, S.-J.; Das, R.R.; Noh, C.; Jin, Y.W. Color tuning of electrochromic materials for Color e-Paper. In Proceedings of the Abstract 2348, 218th ECS Meeting, Lavages, NV, USA, 10-15 October 2010; pp. 2348.

50. Good Display GDE021A1 Specifications.

51. E Ink Holdings Inc. Technical Specification ED060SCE, 2010.

52. Kent Displays Inc. VGA Cholesteric Display Module with SPI Compatible interface Datasheet, 2006.

53. Feenstra, B.J.; Hayes, R.A.; van Dijk, R.; Boom, R.G.H.; Wagemans, M.M.H.; Camps, I.G.; Giraldo, A.; Heijden, B.v.d. Electrowetting-Based Displays: Bringing Microfluidics Alive On-Screen; IEEE: New York, NY, USA, 2006; pp. 48-53. 
54. Hack, M.; Hewitt, R.; Brown, J.J.; Choi, J.W.; Cheon, J.H.; Kim, S.H.; Jang, J. P-11: Analysis of Low Power Consumption AMOLED Displays on Flexible Stainless Steel Substrates; SID Symposium Digest of Technical Papers; Wiley: Hoboken, NJ, USA, 2007; Volume 38, pp. 210-213.

55. Wu, C.; Meng, Z.; Li, J.; Zhang, X.; Yang, G.; Xiong, S.; Shi, X.; Peng, H.; Wong, M.; Kwok, H.S. 35.4: A 2.1-Inch AMOLED Display Based on Metal-Induced Laterally Crystallized Polycrystalline Silicon Technology; SID Symposium Digest of Technical Papers; Wiley: Hoboken, NJ, USA, 2004; Volume 35, pp. 1128-1131.

56. Steudel, S.; Myny, K.; Schols, S.; Vicca, P.; Smout, S.; Tripathi, A.; van der Putten, B.; van der Steen, J.-L.; van Neer, M.; Schütze, F. Design and realization of a flexible QQVGA AMOLED display with organic TFTs. Org. Electron. 2012, 13, 1729-1735.

57. Zhu, F. OLED Activity and Technology Development. In Proceedings of the Symposium on Sustainability Driven Innovative Technologies, Hong Kong, China, 7-8 May 2009.

58. Hack, M.G.; Chwang, A.B.; Lu, M.-H.M.; Kwong, R.C.; Weaver, M.S.; Tung, Y.-J.; Brown, J.J. Flexible low-power-consumption OLED displays for a universal communication device. SPIE 2003, doi:10.1117/12.488783.

59. González, R.A.; Aguilar, P.C.M. Tecnología Oled Y Moled. Vis. Electrón. Algo Más Que Estado Sólido 2011, 4, 34-48.

60. Borchardt, J.K. Developments in organic displays. Mater. Today 2004, 7, 42-46.

61. OLED-Info. OLED Introduction and Basic OLED Information. Available online: http://www.oled-info.com/introduction (accessed on 31 July 2015).

62. Sempel, A.; Büchel, M. Design aspects of low power polymer/OLED passive-matrix displays. Org. Electron. 2002, 3, 89-92.

63. Densitron. Approval Product Specification, C0201QILK-C. Available online: http://datasheet.eeworld.com.cn/pdf/285017,AZDISPLAYS,C0201QILK-C.pdf (accessed on 6 August 2015).

64. U.S. Micro Products Inc. AMOLED USMP-A34480TP Product Specification. Available online: http://www.usmicroproducts.com/sites/default/files/datasheets/USMP-A34480TP_1.pdf (accessed on 6 August 2015).

65. 4D Systems. MicroOLED-160-G2 Display Datasheet. Available online: http://www.4dsystems.com.au/product/1/3/4D_Intelligent_Display_Modules/uOLED_160_G2/ (accessed on 6 August 2015).

66. RitDisplay Corp. Product Specification, RGS32256064WH002. Available online: http://www.gamma.spb.ru/download/P21301-X02.pdf (accessed on 6 August 2015).

67. King, C.N. Electroluminescent Displays. Available online: http://ch00ftech.com/wpcontent/uploads/2012/05/mrsnf98.pdf (accessed on 6 August 2015).

68. Ran, F.; Yang, X.; Huan, X. Design of Thin Film Electroluminescent (TFEL) Display Panel Driver. Adv. Mater. Res. 2012, 462, 45-51.

69. Palalau, S.; Borzea, M.O.; Toffolo, D.; Roza, R.M. Transparent EL Display. U.S. Patent, 6,115,008, 5 September 2000.

70. Lumineq ELT256.120.90 Technical Data Sheet. Available online: http://lumineq.com/sites/default/ files/product/fields/field_product_data_sheet/elt_256.120.90_2.pdf (accessed on 6 August 2015).

71. Heikenfeld, J.C.; Steckl, A.J. Inorganic EL displays at the crossroads. Inf. Disp. 2003, 19, $20-25$. 
72. Planar EL320.240.36-HB High-Bright Small Graphics Display 2009.

73. Sharp LJ32H028 El Display Module Features.

74. Chang, P.-L.; Wu, C.-C.; Leu, H.-J. Investigation of technological trends in flexible display fabrication through patent analysis. Displays 2012, 33, 68-73.

75. Kiyokawa, K. Occlusion Displays. In Handbook of Visual Display Technology; Chen, J., Cranton, W., Fihn, M., Eds.; Springer: Berlin, Germany, 2012; pp. 2251-2257.

76. Choi, M.-C.; Kim, Y.; Ha, C.-S. Polymers for flexible displays: From material selection to device applications. Prog. Polym. Sci. 2008, 33, 581-630.

77. Bae, S.; Kim, H.; Lee, Y.; Xu, X.; Park, J.-S.; Zheng, Y.; Balakrishnan, J.; Lei, T.; Kim, H.R.; Song, Y.I. Roll-to-roll production of 30-inch graphene films for transparent electrodes. Nat. Nanotechnol. 2010, 5, 574-578.

78. Wedding, C.A.; Strbik, O.M., III; Peters, E.F.; Guy, J.; Wedding, D.K. Overview of Flexible Plasma Display Technology. In Proceedings of the ASID '06, New Delhi, India, 8-12 October 2006; pp. 323-337

79. Yoon, C.-D.K.; Hwang, Y.-K.; Chung, I.-J.; Mark, F.; Green, D.; Pangle, M.; McIntyre, J.; Smith, R.D. Recent Progress of Flexible AMOLED Displays. Proc. SPIE 2011, doi:10.1117/12.880144.

80. E Ink Holdings Inc. Mobius, the First Large Format Flexible Display Technology to Go into Mass Production. Available online: http://www.eink.com/press_releases/e_ink_ introduces_mobius_051313.html (accessed on 31 July 2015).

81. Geng, J. A volumetric 3D display based on a DLP projection engine. Displays 2013, 34, 39-48.

82. Dodgson, N.A. Autostereoscopic 3D displays. Computer 2005, 38, 31-36.

83. Dodgson, N.A. Optical devices: 3D without the glasses. Nature 2013, 495, 316-317.

84. Vallerio, K.S.; Zhong, L.; Jha, N.K. Energy-efficient graphical user interface design. Mob. Comput. IEEE Trans. 2006, 5, 846-859.

85. Flinn, J.; Satyanarayanan, M. Energy-aware adaptation for mobile applications. SIGOPS Oper. Syst. Rev. 1999, 33, 48-63.

86. Rabaey, J.M.; Pedram, M. Low Power Design Methodologies; Springer US: Boston, MA, USA, 1996.

87. Anggorosesar, A.; Rim, K.-W.; Kim, Y.-J. A Survey of Low-power Techniques for Liquid Crystal Display Systems with Light Emitting Diode Backlight Units. IETE Tech. Rev. 2011, 28, 351.

88. Cheng, W.-C.; Chao, C.-F. Minimization for LED-backlit TFT-LCDs. In Proceedings of the 43rd Annual Design Automation Conference ACM, New York, NY, USA, 24-28 July 2006; pp. 608-611.

89. Choi, L.; Shim, H.; Chang, N. Low-Power Color TFT LCD Display for Hand-Held Embedded Systems. In Proceedings of the International Symposium on Low Power Electronics and Design, Monterey, CA, USA, 12-14 August 2002; pp. 112-117.

90. Shim, H.; Chang, N.; Pedram, M. A Compressed Frame Buffer to Reduce Display Power Consumption in Mobile Systems. In Proceedings of the ASP-DAC 2004. Asia and South Pacific Design Automation Conference, Yokohama, Japan, 27-30 January 2004; pp. 819-824. 
91. Cheng, W.-C.; Hou, Y.; Pedram, M. Power Minimization in a Backlit TFT-LCD Display by Concurrent Brightness and Contrast Scaling; IEEE Computer Society: Washington, DC, USA, 2004; p. 10252.

92. Dong, M.; Choi, Y.-S.K.; Zhong, L. Power Modeling of Graphical User Interfaces on OLED Displays. In Proceedings of the 46th Annual Design Automation Conference, DAC '09, New York, NY, USA, 26-31 July 2009; pp. 652-657.

93. Zhao, M.; Zhang, H.; Chen, X.; Chen, Y.; Xue, C.J. Online OLED Dynamic Voltage Scaling for Video Streaming Applications on Mobile Devices. In Proceedings of the Ninth IEEE/ACM/IFIP International Conference on Hardware/Software Codesign and System Synthesis, Montreal, QC, Canada, 29 September-4 October 2013; p. 9.

94. Lee, C.; Monga, V. Power-Constrained RGB-to-RGBW Conversion for Emissive Displays. In Proceedings of the 2014 IEEE International Conference on Acoustics, Speech and Signal Processing (ICASSP), Florence, Italy, 4-9 May 2014; pp. 1205-1209.

95. Kansal, A.; Hsu, J.; Zahedi, S.; Srivastava, M.B. Power management in energy harvesting sensor networks. ACM Trans. Embed. Comput. Syst. TECS 2007, 6, 651-656.

96. GBI Research. Energy Efficient Displays Technologies to 2020-OLED Displays Set to Propel Growth of the Industry; GBI Research: London, UK, 2010.

(C) 2015 by the authors; licensee MDPI, Basel, Switzerland. This article is an open access article distributed under the terms and conditions of the Creative Commons Attribution license (http://creativecommons.org/licenses/by/4.0/). 